\title{
Double Structural Transition in Hybrid Material MIL-53 upon Hydrocarbon Adsorption: The Thermodynamics Behind the Scenes
}

\author{
François-Xavier Coudert, Caroline Mellot-Draznieks, Alain H. Fuchs, and Anne Boutin* \\ École Nationale Supérieure de Chimie de Paris (Chimie ParisTech), CNRS and Univ. Pierre et Marie Curie, \\ 11 rue Curie, F-75005 Paris, France, Department of Chemistry, UCL, 20 Gordon Street, \\ London, WC1H OAJ, United Kingdom, and Laboratoire de Chimie Physique, CNRS and Univ. Paris-Sud, Bât. 349, \\ F-91405 Orsay Cedex, France
}

Received December 9, 2008; E-mail: anne.boutin@Icp.u-psud.fr

In this paper, we rationalize the thermodynamics behind the guest-induced structural transitions of hybrid material MIL-53, showing that the existence of a double transition depends on the relative guest affinities for the two phases, rather than absolute adsorption enthalpies. Based on a simple model, we interpret recent experimental data on alkane adsorption and predict transition pressures.

Porous metal-organic frameworks (MOFs) are a topical class of materials that display an extremely large range of crystal structures and host-guest properties, potentially giving them a major impact in adsorption, separation, and storage of strategic gases $\left(\mathrm{H}_{2}, \mathrm{CO}_{2}, \mathrm{CH}_{4}, \ldots\right)$. A growing number of these materials show exceptional guest-responsive behaviors upon gas adsorption, due to the flexibility of their organic-inorganic frameworks. This includes examples of progressive swelling or contraction (also called breathing), pore deformation, and amorphous-to-crystal and crystalto-crystal structural transitions. ${ }^{1}$ The MIL-53 materials family, ${ }^{2}$ a particularly eye-catching case of the last category, has attracted a lot of attention due to its large flexibility and the occurrence of a double structural transition upon adsorption of some gases $\left(\mathrm{CO}_{2}\right.$, $\left.\mathrm{H}_{2} \mathrm{O}, \mathrm{C}_{2} \mathrm{H}_{6}, \ldots\right)$ but not others $\left(\mathrm{H}_{2}, \mathrm{CH}_{4}\right){ }^{3}$ It was also reported very recently ${ }^{4}$ that liquid phase adsorption of para-xylene does induce a structural transition, while ortho- and meta-xylene do not. The studies performed so far, both experimentally and by molecular simulation, mainly focused on structural characterization and energetics (by calorimetry, forcefield-based calculations and DFT). However, the current depiction of these guest-induced structural transitions is lacking a general thermodynamic interpretation of all the results obtained so far; Llewellyn et al. indeed highlighted the necessity of understanding "the thermodynamic conditions for the host-guest interactions which allow the breathing to happen". 3

We recently developed a generic thermodynamic framework for the understanding of guest-induced structural transitions in flexible nanoporous materials such as MOFs, ${ }^{5}$ by use of the osmotic pseudoensemble. For a material that has two possible framework structures and where gas adsorption follows type I isotherms, ${ }^{6}$ we proposed a full taxonomy of possible guest-induced structural transitions. This classification relies only on a few key parameters, such as the free energy difference, $\Delta F$, between the (empty) host structures, their pore volumes, $V_{\mathrm{p}}{ }^{(i)}$, and the adsorption affinities for the guest, $K_{i}$. This method also allows us to calculate one of these parameters when the pressures of structural transitions are known. The robustness of the method was demonstrated on systems exhibiting such contrasting behaviors as "breathing" and "gate opening". In the case of MIL-53 (Al), in particular, we used the available $\mathrm{CO}_{2}$ adsorption isotherm to calculate a free energy difference between the empty large pore (lp, Figure 1S) and narrow pore (np, Figure $2 \mathrm{~S}$ ) forms of $\Delta F \approx 2.5 \mathrm{~kJ} / \mathrm{mol}$. This value was
Table 1. Predicted Pressures for the $I \mathbf{p} \rightarrow \mathrm{np}$ and $\mathrm{np} \rightarrow \mathrm{Ip}$ Transitions

\begin{tabular}{ccccc}
\hline guest & $\mathrm{CO}_{2}$ & $\mathrm{C}_{2} \mathrm{H}_{6}$ & $\mathrm{C}_{3} \mathrm{H}_{8}$ & $\mathrm{C}_{4} \mathrm{H}_{10}$ \\
\hline$P(\mathbf{l p} \rightarrow \mathbf{n p})$ & $0.3 \mathrm{bar}$ & $0.17 \mathrm{bar}$ & $30 \mathrm{mbar}$ & $9.0 \mathrm{mbar}$ \\
$P(\mathbf{n p} \rightarrow \mathbf{l p})$ & $5 \mathrm{bar}$ & $3.3 \mathrm{bar}$ & $0.45 \mathrm{bar}$ & $0.15 \mathrm{bar}$ \\
\hline
\end{tabular}

then used to successfully predict the position of the low-pressure $\mathbf{l p} \rightarrow \mathbf{n p}$ transition, confirmed by calorimetry. ${ }^{7}$ This rather small value of $2.5 \mathrm{~kJ} / \mathrm{mol}$ is of the order of $k T$ at room temperature, explaining the bistability of MIL-53 (Al). Indeed, all guest-responsive hybrid materials we studied so far exhibit free energy differences between host structures in the range $2-5 \mathrm{~kJ} / \mathrm{mol}$. This is in sharp contrast with the hypothesis of Llewellyn et al. that $\Delta F$ should be much greater than $k T,{ }^{3}$ but not incompatible with the vision of Zhang and Chen that the energy barrier between different structures has to be much larger than $k T$ for materials where flexibility is thermodynamically controlled. ${ }^{8}$

We now turn to the issue of how the presence or absence of guest-induced structural transitions can be related to the properties of the guest molecule. New experimental data on the adsorption of various species in the pores of MIL-53 have been published very recently, in both gas phase (for $\mathrm{C}_{n} \mathrm{H}_{2 n+2}, n \leq 4$ ) ${ }^{3}$ and liquid phase (for $o-, m$-, and $p$-xylene). ${ }^{4}$ In the case of materials such as MIL53, where the large-pore (lp) form is intrinsically more stable than the narrow-pore (np) form at room temperature, our taxonomy predicts either the occurrence of two structural transitions upon gas adsorption or the absence of any transition. This is determined by a balance between intrinsic stability of the crystal structures, adsorption affinities, and accessible volume. In the case of alkanes adsorption in MIL-53, adsorption isotherms from ref 3 show that the variation of pore volume (and adsorbed quantities at saturation) for both phases is small and the main factor is the change in adsorption affinities for different guests (see Table 1S). Within the limits of this model, the presence or absence of structural transitions for a given adsorbate is unambiguously determined by one factor only: the ratio $K_{\mathrm{np}} / K_{\mathrm{lp}}$ of adsorption affinities in the two structures. A double guest-induced structural transition happens if and only if $K_{\mathrm{lp}} / K_{\mathrm{np}}$ is large enough so that the following inequality holds: ${ }^{5}$

$$
\begin{aligned}
& \left(V_{\mathrm{p}}^{(\mathbf{n p})}-V_{\mathrm{p}}^{(\mathbf{l p})}\right) \ln \left(\frac{K_{\mathrm{np}} / K_{\mathrm{lp}} \times V_{\mathrm{p}}^{(\mathbf{l p})}-V_{\mathrm{p}}^{(\mathbf{n p})}}{V_{\mathrm{p}}^{(\mathbf{l p})}-V_{\mathrm{p}}^{(\mathbf{n p})}}\right)+ \\
& \qquad V_{\mathrm{p}}^{(\mathbf{l p})} \ln \frac{K_{\mathrm{np}}}{K_{\mathrm{lp}}} \geq \frac{\Delta F}{\rho R T}
\end{aligned}
$$

If, on the contrary, $K_{\mathrm{lp}} / K_{\mathrm{np}}$ is too small, there will be no structural transition at all. This conclusion that the existence of a double structural transition depends on the relative affinities of the 


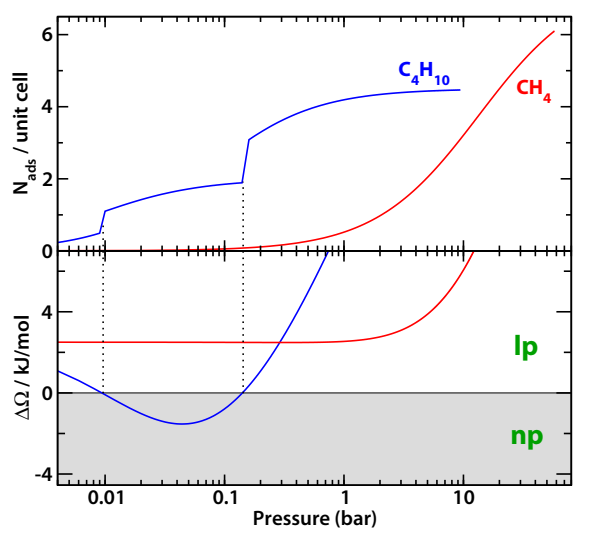

Figure 1. Upper panel: adsorption isotherms of $\mathrm{CH}_{4}$ (in red) and $\mathrm{C}_{4} \mathrm{H}_{10}$ (in blue) in MIL-53 (Cr), in a Langmuir model. Lower panel: difference in osmotic potential between $\mathbf{l p}$ and $\mathbf{n p}$ phases, as a function of pressure. Vertical dotted lines correspond to $\mathrm{C}_{4} \mathrm{H}_{10}$-induced structural transition.

adsorbate in both structures is at variance with the view that the double transition is dictated solely by the affinity for the lp form (with a proposed critical adsorption enthalpy of $\sim 20 \mathrm{~kJ} / \mathrm{mol}$ ). ${ }^{3}$ Our findings are directly supported by very recent experiments showing that, although affinities of xylene isomers for MIL-53 (Al) follow the order ortho $>$ meta $>$ para, only para-xylene induces a double structural transition upon adsorption. ${ }^{4}$

By way of illustration, we show in Figure 1 the adsorption isotherms of $\mathrm{C}_{4} \mathrm{H}_{10}$ and $\mathrm{CH}_{4}$ in MIL-53 $(\mathrm{Cr})$ at $303 \mathrm{~K}$, using for each structure (lp and np) Langmuir isotherms fitted on experimental data $^{3}$ (the parameters are detailed in the Supporting Information). For $\mathrm{C}_{4} \mathrm{H}_{10}, K_{\mathrm{np}} / K_{\mathrm{lp}} \approx 4$ and there are two successive structural transitions ( $\Delta \Omega$ changes sign twice); this leads to an isotherm where two steps can be clearly seen on a logarithmic pressure scale. Contrarily, for $\mathrm{CH}_{4}, K_{\mathrm{np}} / K_{\mathrm{lp}} \approx 1$ and the lp structure is thermodynamically favored throughout the pressure range; the isotherm, being simply that of $\mathrm{CH}_{4}$ in MIL-53 lp, has no step.

Finally, we focus on the linear relationship observed by Llewellyn et al. between the $\mathbf{n p} \rightarrow \mathbf{l p}$ transition pressure and the adsorption enthalpies of gases in the $\mathbf{n p}$ and $\mathbf{l p}$ structures. To understand this, we study the evolution of the pressure at which structural transitions happen in the material as a function of $K_{\mathrm{np}}$ and $K_{\mathrm{lp}}$. To relate these adsorption affinities to adsorption enthalpies and entropies, we write the following:

$$
K_{i}=A \exp \left(-\Delta H_{i} / R T\right) \exp \left(\Delta S_{i} / R\right)
$$

We can then show (see Supporting Information) that the pressure of both the $\mathbf{n p} \rightarrow \mathbf{l p}$ and $\mathbf{l p} \rightarrow \mathbf{n p}$ structural transitions exhibits a linear $\log (P)$ vs $\Delta H$ relation, if the $K_{\mathrm{np}} / K_{\mathrm{lp}}$ ratio is constant. By virtue of eq 2 , this condition translates to $\Delta \Delta H=\Delta H_{\mathrm{np}}-\Delta H_{\mathrm{lp}}$ and $\Delta \Delta S$ $=\Delta S_{\mathrm{np}}-\Delta S_{\mathrm{lp}}$ being constant among a series of adsorbates. While the first equality for alkane adsorption in MIL-53 (Cr) can be verified from the Monte Carlo results presented in ref 3, we suggest that the second is a consequence of the often observed linear relationship between $\Delta H_{\text {ads }}$ and $\Delta S_{\text {ads }}$ for adsorption of alkanes in microporous materials. ${ }^{9}$ We thus conclude that the logarithmic relationship between $P$ and adsorption enthalpies is not a universal feature but a direct consequence of a constant difference between adsorption enthalpies in both forms of MIL-53. Furthermore, we predict that the pressure of the $\mathbf{l} \mathbf{p} \rightarrow \mathbf{n p}$ transition follows this same logarithmic relationship, enabling us to predict transition pressures

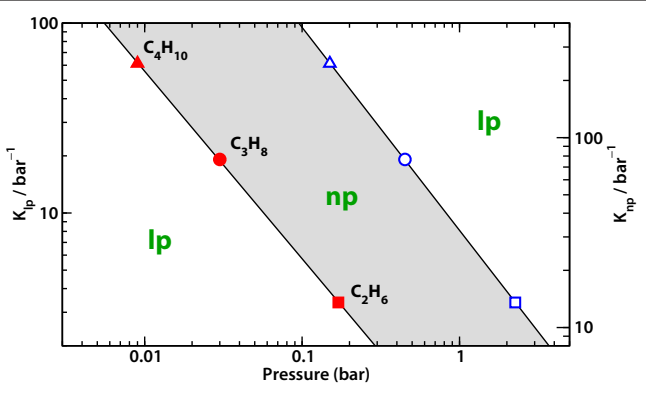

Figure 2. Existence domains of the $\mathbf{l p}$ and $\mathbf{n p}$ phases of MIL-53 upon gas adsorption, with guests of different affinities $\left(K_{\mathrm{lp}}\right.$ and $\left.K_{\mathrm{np}}\right)$. Symbols correspond to $\mathbf{l} \mathbf{p} \rightarrow \mathbf{n p}$ and $\mathbf{n p} \rightarrow \mathbf{l p}$ (respectively, filled and open) transitions for the $\mathrm{C}_{2}$ to $\mathrm{C}_{4}$ alkanes.

that have not been experimentally measured yet. Finally, we can draw a phase diagram (Figure 2) of MIL-53 (Cr) as a function of guest pressure and host-guest affinities, for a series of adsorbates with a constant $K_{\text {lp }} / K_{\text {np }}$ ratio. We show on this phase diagram the points corresponding to the phase transitions induced by $\mathrm{C}_{2} \mathrm{H}_{6}, \mathrm{C}_{3} \mathrm{H}_{8}$, and $\mathrm{C}_{4} \mathrm{H}_{10}$. It is noteworthy that the pressure domain of the $\mathbf{n p}$ phase, which has a constant width in the logarithmic scale of Figure 2 , actually gets smaller in a linear scale for adsorbates with larger affinities for the solid. Figures $3 \mathrm{~S}$ and $4 \mathrm{~S}$ show additional 2D and $3 \mathrm{D}$ cuts in the phase diagram as described by our model.

In conclusion, we have elucidated and rationalized the thermodynamics of the "breathing" phenomenon of MIL-53, highlighting the case of hydrocarbon adsorption. We demonstrated that the existence of the breathing is determined by the relative affinities of the sorbate for the lp and $\mathbf{n p}$ phases and proposed an explanation for the linear $\log (P)$ vs $\Delta H_{\text {ads }}$ relation observed by Llewellyn et al. $^{3}$

Acknowledgment. This work was supported by the EPSRC (Advanced Research Fellowship awarded to CMD) and the FP-6 European funding STREP “SURMOF” (NMP4-CT-2006-032109).

Supporting Information Available: Calculation details, structures of MIL-53 (Cr) in lp and np form, and 2D representations of the solutions of eq 1 . This material is available free of charge via the Internet at http://pubs.acs.org.

\section{References}

(1) Kitagawa, S.; Uemura, K. Chem. Soc. Rev 2005, 34, 109. Fletcher, A. J.; Thomas, K. M.; Rosseinsky, M. J. J. Solid State Chem. 2005, 178, 2491. Mellot-Draznieks, C.; Serre, C.; Surblé, S.; Audebrand, N.; Férey, G. J. Am. Chem. Soc. 2005, 127, 16273. Serre, C.; Mellot-Draznieks, C.; Surblé, S.; Audebrand, N.; Filinchuk, Y.; Férey, G. Science 2007, 315, 1828.

(2) Millange, F.; Serre, C.; Férey, G. Chem. Commun. 2002, 822. Serre, C.; Millange, F.; Thouvenot, C.; Nogues, M.; Marsolier, G.; Louër, D.; Férey, G. J. Am. Chem. Soc. 2002, 124, 13519. Liu, Y.; Her, J.-H.; Dailly, A.; Ramirez-Cuesta, A. J.; Neumann, D. A.; Brown, C. M. J. Am. Chem. Soc. 2008, 130, 11813.

(3) Llewellyn, P. L.; Maurin, G.; Devic, T.; Loera-Serna, S.; Rosenbach, N.; Serre, C.; Bourrelly, S.; Horcajada, P.; Filinchuk, Y.; Férey, G. J. Am. Chem. Soc. 2008, 130, 12808 .

(4) Alaerts, L.; Maes, M.; Giebeler, L.; Jacobs, P. A.; Martens, J. A.; Denayer, J. F. M.; Kirschhock, C. E. A.; De Vos, D. E. J. Am. Chem. Soc. 2008, 130, 14170.

(5) Coudert, F.-X.; Jeffroy, M.; Fuchs, A. H.; Boutin, A.; Mellot-Draznieks, C. J. Am. Chem. Soc. 2008, 130, 14294.

(6) Rouquerol, F.; Rouquerol, J.; Sing, K. Adsorption by Powders and Porous Solids; Academic Press: New York, 1999.

(7) Bourrelly, S.; Llewellyn, P. L., private comunication.

(8) Zhang, J.-P.; Chen, X.-M. J. Am. Chem. Soc. 2008, 130, 6010.

(9) Wender, A.; Barreau, A.; Lefebvre, C.; Di Lella, A.; Boutin, A.; Ungerer, P.; Fuchs, A. H. Adsorption 2007, 13, 439. Atkinson, D.; Curthoys, G. J. Chem. Soc., Faraday Trans. 1 1981, 77, 897.

JA8094153 